\title{
Clinical Benefit Response in Pancreatic Cancer Trials Revisited
}

\author{
Jürg Bernhard ${ }^{a, b}$ Daniel Dietrich ${ }^{a}$ Bengt Glimelius ${ }^{c}$ György Bodoky ${ }^{d}$ \\ Werner Scheithauer ${ }^{\text {eff }}$ Richard Herrmann ${ }^{g}$
}

aSAKK Coordinating Center, Bern; 'bepartment of Medical Oncology, Inselspital, Bern University Hospital, Switzerland; 'Department of Radiology, Oncology and Radiation Science, Uppsala University/Akademiska sjukhuset, Sweden; 'Department of Oncology, St. László Hospital, Budapest, Hungary; ${ }^{e}$ Department of Internal Medicine I and CCC, Medical University Vienna; ${ }^{\mathrm{f}}$ Central European Cooperative Oncology Group, Vienna, Austria; ${ }^{9}$ Department of Clinical Research, University Hospital Basel, Switzerland

\section{Keywords}

Clinical benefit response - Patient-reported outcome . Quality of life - Palliation · Pancreatic carcinoma, advanced

\section{Summary}

Objectives: Clinical benefit response (CBR), based on changes in pain, Karnofsky performance status, and weight, is an established palliative endpoint in trials for advanced gastrointestinal cancer. We investigated whether CBR is associated with survival, and whether CBR reflects a wide-enough range of domains to adequately capture patients' perception. Methods: CBR was prospectively evaluated in an international phase III chemotherapy trial in patients with advanced pancreatic cancer ( $n=311$ ) in parallel with patient-reported outcomes (PROs). Results: The median time to treatment failure was 3.4 months (range: $0-6$ ). The majority of the CBRs $(n=39$ ) were noted in patients who received chemotherapy for at least 5 months. Patients with CBR ( $n=62$ ) had longer survival than non-responders ( $n=182$ ) (hazard ratio $=0.69 ; 95 \%$ confidence interval: $0.51-0.94 ; p=0.013)$. CBR was predicted with a sensitivity and specificity of $77-80 \%$ by various combinations of 3 mainly physical PROs. A comparison between the duration of $\mathrm{CBR}$ ( $n=62$, median $=8$ months, range $=4-31$ ) and clinically meaningful improvements in the PROs ( $n=100-116$; medians $=9-11$ months, range $=4-24$ ) showed similar intervals. Conclusion: CBR is associated with survival and mainly reflects physical domains. Within phase III chemotherapy trials for advanced gastrointestinal cancer, CBR can be replaced by a PRO evaluation, without losing substantial information but gaining complementary information.

\section{Introduction}

In a recent commentary, Booth and colleagues [1] called for clarity in the reporting of benefit associated with anticancer therapies. They suggested that the use of clinical benefit should be restricted to patient-centered outcomes, in accordance with the original definition by Rothenberg et al. [2] and Burris et al. [3], as opposed to tumor-centered outcomes. Rothenberg, Burris and colleagues introduced clinical benefit response (CBR) in the mid 1990s for clinical trials in pancreatic cancer as a composite endpoint of changes in pain, Karnofsky performance status (KPS), and weight to be observed for at least 4 consecutive weeks.

The concept of CBR has been criticized from the very beginning. According to Gelber [4], perhaps the most difficult issue in defining net patient benefit is that the quality of life (QoL) it seeks to evaluate is confounded with the duration of survival available to the patient. Furthermore, he questioned whether evaluations based on pain relief and performance status alone reflect a wide-enough range of domains to capture adequately the patients' assessment of their QoL [4]. Hoffman and Glimelius [5] retrospectively applied CBR criteria in 2 randomized trials in upper gastrointestinal cancer and compared them to the physicians' 'subjective response' evaluation and the patients' standardized QoL assessment. CBR overestimated the beneficial effects in certain patients and underestimated them in others, mainly due to its limitations in reflecting important aspects influencing patients' well-being (e.g. side effects of chemotherapy, other tumor symptoms) [5].

There are practical limitations in evaluating CBR in clinical trials. The daily assessment of pain and analgesic consumption, as part of the original definition of CBR, is demanding for the patients and hardly suitable over several months [6]. Missing data are well known when patient diaries are used [7]. Due to its hierarchical structure, the algorithm of CBR is particularly 
vulnerable to missing data and, thus, the loss of evaluable cases.

Irrespective of such criticism, CBR based on either patientcentered or tumor-centered definitions has been increasingly used as a trial endpoint in various cancer sites [8]. To our knowledge, the potential confounding of CBR with survival has never been addressed. With a few exceptions [5, 6, 9], this development has been independent from the establishment of QoL or other patient-rated outcomes (PROs) in cancer clinical trials. In particular, the value of changes in analgesic consumption, KPS, or weight for the evaluation of a palliative treatment, as compared to patients' perception, has not been investigated conclusively.

In an international phase III chemotherapy trial in patients with advanced pancreatic cancer $[6,10]$, we prospectively investigated whether CBR is associated with survival; and whether CBR reflects a wide-enough range of domains to capture adequately patients' perception, or vice versa, whether PROs may cover the information derived from the complex algorithm of CBR.

\section{Patients and Methods}

\section{The Trial}

All patients with histologically proven, locally advanced or metastatic adenocarcinoma of the pancreas, treated in the international phase III trial SAKK 44/00-CECOG/PAN.1.3.001 [10], were included in this study. Patients had a KPS $>60$, were naïve to chemotherapy for advanced disease, and had not received any adjuvant radiotherapy or radio-chemotherapy 12 months prior to inclusion. Patients were stratified by KPS (90-100 vs. 60-80), disease extent (locally advanced vs. metastatic), presence or absence of pain, and by enrolling center. Patients were then randomly assigned to GemCap (oral capecitabine $650 \mathrm{mg} / \mathrm{m}^{2}$ twice daily on days 1-14 plus gemcitabine $1,000 \mathrm{mg} / \mathrm{m}^{2} 30-\mathrm{min}$ infusion days 1 and 8 every 3 weeks) or Gem (gemcitabine $1,000 \mathrm{mg} / \mathrm{m}^{2} 30$-min infusion weekly for 7 weeks, followed by a 1-week break, and then weekly for 3 weeks every 4 weeks). Treatment was continued until disease progression or for a maximum of 24 weeks, except in the case of unacceptable toxicity. Treatment could be resumed later at the discretion of the investigator. Treatment decisions were based on clinical and radiographic grounds. Informed consent was obtained from all patients, and ethics committee approval was given by all participating centers. Trial protocol and conduct are described elsewhere [10].

CBR and PROs in terms of selected QoL indicators were secondary endpoints. Inclusion criteria included a baseline assessment by the patient prior to randomization, with a diary being completed for 4 days at home, and a QoL form filled in at the hospital. Patients were asked to complete a weekly diary (CBR evaluation) for 24 weeks at home, starting at randomization, and a QoL form weekly at the hospital, for the first 7 weeks and subsequently before each administration of Gem for 24 weeks from randomization. This weekly/fortnightly schedule was selected to minimize the potential bias associated with early withdrawal from study treatment and to investigate patients' underlying trajectories of palliation [6]. To avoid any interference with the standard assessment of CBR, the QoL form had to be completed at the hospital, prior to diagnostic procedures. The diary and the QoL form were translated by a 'forward-backward' procedure to obtain conceptual equivalence for the main languages in the participating centers (Finnish, French, German, Hebrew, Hungarian, Italian, and Swedish).

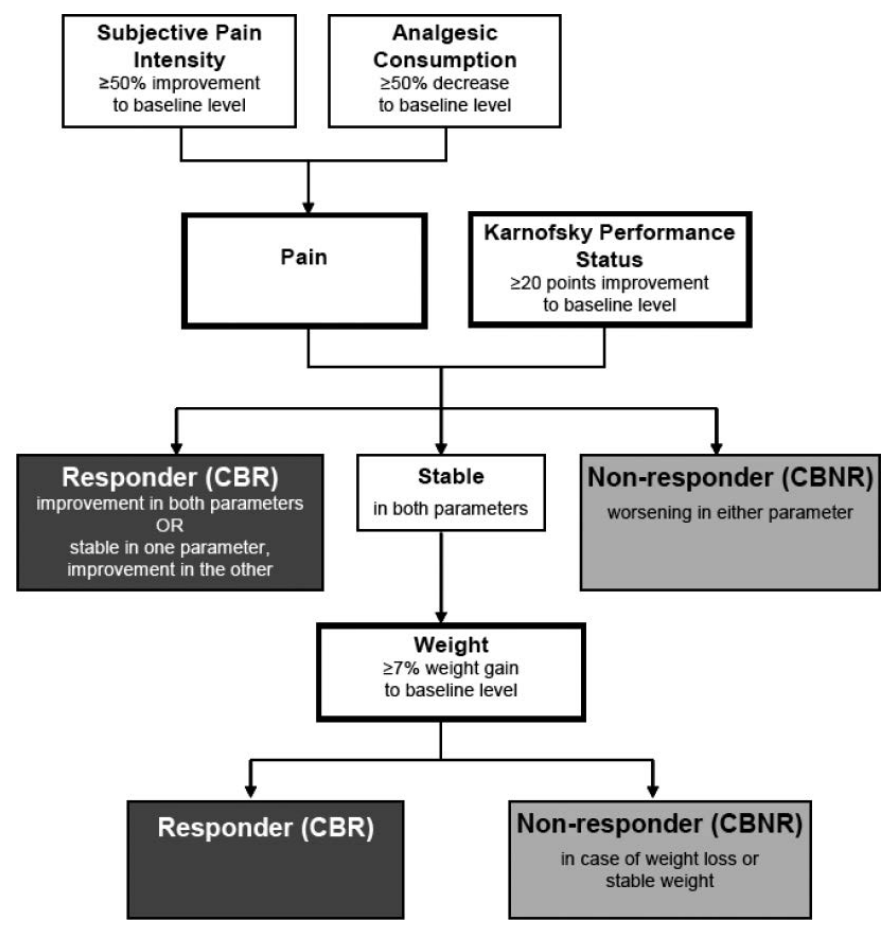

Fig. 1. Definition of clinical benefit response (CBR).

\section{Clinical Benefit Response}

CBR was assessed according to the definition by Burris et al. [3], consisting of pain and KPS as primary criteria, and weight as secondary criterion (fig. 1). Patients rated their pain on a linear-analogue self-assessment (LASA) indicator [11] and documented their use of analgesics daily. An improvement in pain required either a positive change in both pain intensity and analgesic consumption, or a positive change in either factor and a stable situation in the other. Stable pain required a stable situation in both factors. A worsening in pain was defined by a negative change in one or both factors. A CBR required either an improvement in both pain and KPS, or an improvement in either factor and a stable situation in the other. A non-response was defined by a worsening in one or both factors. For patients with a stable situation in both factors, a CBR required a positive change in weight. A non-response was defined by any other result. If evaluable, each patient was determined to have either a CBR or non-response.

\section{Patient Rated Outcomes}

To keep a weekly/fortnightly schedule in addition to the diary feasible, we focused on key aspects of palliation by chemotherapy. We chose LASA indicators that are suitable for the investigation of longitudinal patterns based on short assessment intervals. The following global indicators were used: Overall treatment burden [12], physical well-being [13], mood $[13,14]$, coping effort $[13,15]$ and functional performance [16]. The discriminating capacity of the treatment burden indicator regarding chemotherapy side effects has been shown in patients with advanced gastric carcinoma [17]. The indicators for physical well-being, mood, coping effort, and functional performance were sensitive to tumor response in metastatic colorectal cancer [18]. Psychological distress in pancreatic cancer [19] was assessed by the mood and coping indicators, which are sensitive to mood disorders and psychosocial dysfunction [20]. Responses on these indicators are expected to reflect the summation of the individual meaning and importance of various factors to each patient, resulting in a comparable responsiveness to treatment effects as compared to multiitem scales [20]. In addition, specific indicators for pain [11] (same as for 
CBR) and tiredness [16] were included. All indicators had a range 0-100, with higher scores indicating a better condition. A mean change of $\geq 8$ points from baseline was defined as clinically meaningful [21].

\section{Statistical Analyses}

The association between CBR and survival was analyzed first by Kaplan-Meier curves and log-rank tests to investigate the association between CBR and the available information at the end of follow-up; second by Cox regressions stratified by treatment duration (grouped in 4-week periods) to investigate this association within patient groups who received treatment for the same duration; and third by Cox regressions dependent on treatment duration to investigate the prognostic meaning of this association. Prognostic factors for CBR were analyzed in a generalized logits model. The means of QoL of the patients with a CBR and of the nonresponders were plotted over time to visualize the associations with CBR The time scale of the QoL measurements was divided into successive 4-week periods. Multiple measurements per patient within a 4-week period were averaged. Baseline measurements were included as time ' 0 '. QoL forms filled in $>3$ days before or after day 1 of a cycle were excluded. We report means of untransformed data with $95 \%$ confidence intervals (CIs). To clarify whether QoL indicators can predict CBR, receiver operating characteristic (ROC) curves from logistic regression models with $\mathrm{CBR} /$ non-responders as outcome and $\mathrm{QoL}$ indicators as predictors were calculated and displayed. Baseline QoL measurements, treatment duration in months $(0-1,1-2, \ldots, 5-6)$, and summary measures of QoL on treatment as medians and high quantiles were included in the model Summary measures were modeled within treatment duration to account for interactions with treatment duration. Analyses were performed with SAS 9.2 (SAS Institute Inc., Cary, NC, USA).

\section{Results}

\section{Sample Description and Patient Characteristics}

Of the 319 randomized patients, 4 did not receive study treatment and another 4 patients had no visit forms. Of the 311 eligible patients, 307 (97\%) had a baseline diary and 305 (96\%) a baseline QoL form. Of all expected diaries and QoL forms under treatment, we received $94 \%(4,414$ of 4,655$)$ and $86 \%(3,033$ of 3,536), respectively. Participants and non-participants at the last scheduled QoL (week 23) or diary (week 24) assessment, respectively, were similar regarding age, sex, disease status, KPS, and pain requiring medication at randomization. The assessable CBR and QoL data are summarized in table 1.

At baseline, a majority of patients had metastatic disease and pain requiring analgesic medication (table 2). There were no significant effects between the randomly assigned treatments on survival, CBR, or any of the QoL endpoints $[6,10]$.

\section{Palliative Endpoints and Survival}

Of the 311 patients, 62 had a CBR (20\%), 182 were nonresponders $(59 \%)$, and $67(22 \%)$ were not assessable due to missing data in 1 or more factors of CBR. The median time to treatment failure was 3.4 months (range: $0-6$ ). The majority of the CBRs $(n=39)$ were in patients who received chemotherapy for at least 5 months.
Table 1. Clinical benefit and quality of life data of eligible patients

Table 2. Baseline characteristics of the eligible patients

\begin{tabular}{llllr}
\hline & Clinical benefit & & Total \\
\cline { 2 - 4 } & Responder & Non-responder & Not assessable & \\
\hline Total, $\mathrm{n}$ & 62 & 182 & 67 & 311 \\
Quality of life, $\mathrm{n}$ & & & 3 & 6 \\
$\quad$ No baseline form & 1 & 2 & 10 & 11 \\
$\quad$ Only baseline form & 0 & 179 & 54 & 294 \\
$\quad$ Baseline and treatment forms available* & 61 & & & \\
\hline *Missing items on completed forms are not considered. &
\end{tabular}

\begin{tabular}{|c|c|c|}
\hline & Median & Range \\
\hline Age, years & 62 & $27-84$ \\
\hline \multirow[t]{2}{*}{ Average baseline pain intensity over 4 days $(n=301)^{*}$} & 17 & $0-92$ \\
\hline & Patients, $\mathrm{n}$ & $\%$ \\
\hline Total & 311 & \\
\hline Male/female & $165 / 146$ & $53 / 47$ \\
\hline \multicolumn{3}{|l|}{ Disease } \\
\hline Locally advanced & 65 & 21 \\
\hline \multirow{2}{*}{\multicolumn{3}{|c|}{ Karnofsky performance status $* *$}} \\
\hline & & \\
\hline $90-100$ & 163 & 52 \\
\hline $60-80$ & 148 & 48 \\
\hline \multicolumn{3}{|l|}{ Pain requiring medication $* *$} \\
\hline Present & 210 & 68 \\
\hline Absent & 101 & 32 \\
\hline
\end{tabular}



The association between CBR and survival is illustrated by Kaplan-Meier curves in figure 2; it should be noted that this is not a prognostic analysis for CBR during treatment. Patients with a CBR had better survival than the non-responders (hazard ratio $(\mathrm{HR})=0.69 ; 95 \% \mathrm{CI}: 0.51-0.94 ; \mathrm{p}=0.013)$. Patients who were not assessable for CBR had worse survival than the non-responders $(\mathrm{HR}=1.23$; 95\% CI: 0.91-1.65). This sample also included patients in a good baseline condition, with scores in pain, analgesic consumption, and KPS higher than the threshold for a potential improvement. These patients could be classified as achieving a CBR only if these values remained stable for $\geq 4$ weeks (fig. 1). The association between CBR and survival was also investigated in the subset of 211 patients who could potentially improve in 1 or more of the

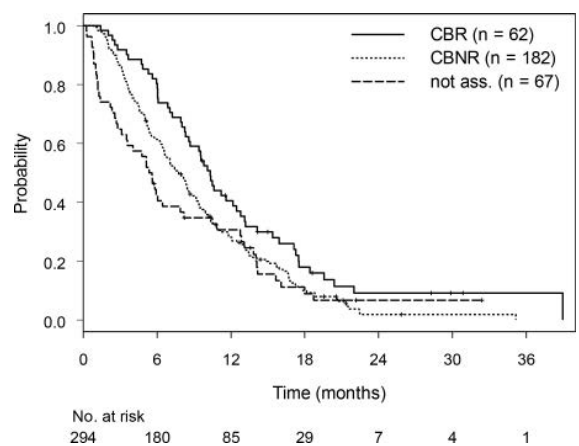

Fig. 2. Association between CBR, no response (CBNR), not assessable response, and overall survival. Kaplan-Meier curves are shown.

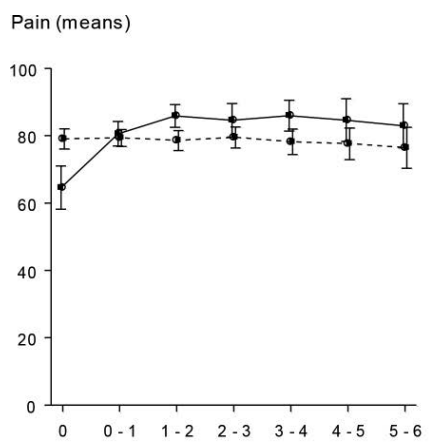

Functional performance (means)

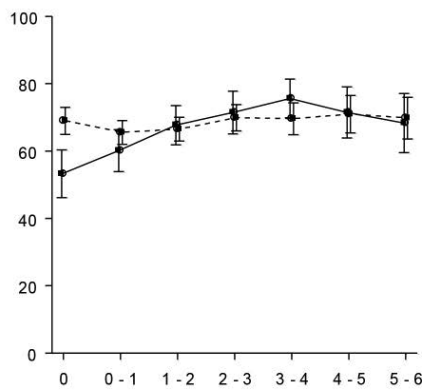

Tiredness (means)

Mood (means)
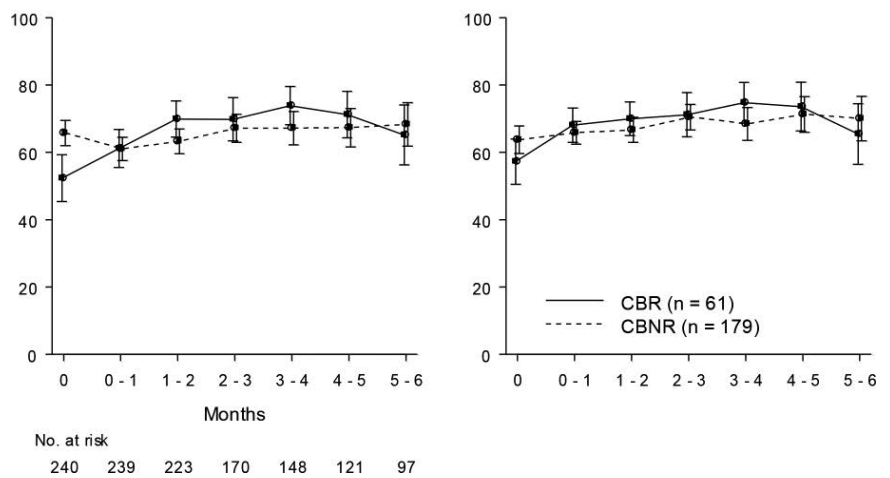

Fig. 3. Pain, functional performance, tiredness, and mood by CBR versus no response (CBNR). Higher scores indicate a better condition for all indicators (scale range: all 0-100). primary CBR criteria. The findings were consistent (data not shown).

Both the CBR rate and survival tended to increase with treatment duration. In an analysis stratified by treatment duration, there was no significant association between CBR and overall survival ( $\mathrm{p}=0.7$ for responders vs. non-responders; $\mathrm{p}=0.3$ including the not-assessable cases as third group). This was confirmed by a time-dependent Cox analysis.

CBR was predicted by patients' baseline status. Patients with pain requiring analgesic medication had a $2.9 \times$ higher chance for a CBR compared to those with pain not requiring analgesics (95\% CI: 1.2-6.6; $\mathrm{p}=0.029)$. Patients with a KPS of 60-80 had a $2.0 \times$ higher chance for a CBR compared to those with 90-100 (95\% CI: 1.0-3.8; p = 0.05). There was no association between baseline tumor marker CA 19-9 and CBR.

\section{CBR and Patients' Perception}

There was a consistent pattern of how patients perceived a CBR: They indicated a worse QoL before starting chemotherapy compared to the non-responders, and a rapid change toward better scores within the first month, as shown in figure 3. The strongest discrimination was before starting chemotherapy, with a mean difference ranging from 6.5 for mood to 15.8 for functional performance, and no discrimination of treatment burden.

We investigated whether these indicators covered the information derived from the $\mathrm{CBR}$ algorithm using logistic regressions and ROC curves. We used baseline scores of all indicators, the number of months with a QoL assessment (corresponding to the time on protocol chemotherapy), and, within this interval, the medium scores on chemotherapy as predictors for CBR. This resulted in a statistically significant model $(p<0.0001)$. The optimal cut-point had a sensitivity (i.e. probability of a correct CBR) and a specificity (i.e. probability of a correct non-response) of $89 \%$. Only baseline scores had a significant impact on predicting CBR (pain: $\mathrm{p}<0.003$; coping effort: $\mathrm{p}<0.005$ ). In a backward elimination, pain was the only significant factor during treatment $(\mathrm{p}<0.007)$.

Further summary measures of QoL scores on chemotherapy were investigated. The maximum values on treatment, in addition to the medians, had a marginal impact on the prediction of CBR. To give more weight to the time that these patients reported high QoL scores, we included both the median and the $90 \%$ quantile of all assessments during treatment. Using all indicators, CBR could then be predicted with a sensitivity and specificity of over $90 \%$ and an area under the curve (AUC) of 0.975 (fig. 4).

The main effect of months with a QoL assessment was between a follow-up of up to 5 months and more than 5 months. We reduced the number of time patterns accordingly to a binary predictor. We further simplified the model using combinations of only 3 indicators. These combinations were composed of 3 of the following indicators: pain, functional 


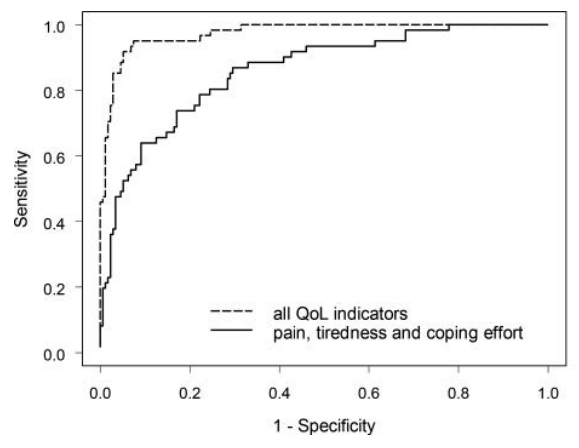

Fig. 4. Receiver operating characteristic curves from logistic regression models with CBR (yes/no) as outcome, and quality of life (QoL) indicators as predictors (models described in the results).

performance, physical well-being, tiredness, or coping effort. They resulted in a sensitivity and specificity of $77-80 \%$. The example of pain, functional performance, coping effort had an AUC of 0.857 (fig. 4).

A comparison between the duration of CBR $(n=62$, median $=8$ months, range $=4-31$ ) and the duration of a clinically meaningful improvement in the QoL indicators, for at least 4 consecutive weeks, showed similar intervals. The longest improvement was in coping effort $(n=116$, median $=$ 11 months, range $=4-24)$, the shortest in functional performance $(\mathrm{n}=100$, median $=9$ months, range $=4-24)$.

\section{Discussion}

Patients with longer survival and thus with longer trial treatment, in particular those with at least 5 months, were more likely to experience a CBR. CBR was predicted by baseline pain requiring analgesics and by a lower KPS, thus CBR was more likely in patients who could potentially improve. The majority of patients had a shorter treatment than 5 months. The palliation by chemotherapy was less obvious for those patients. For example, patients who did not have a CBR could potentially be stable in the factors of CBR and improve in additional symptoms not included in this definition (e.g. psychological distress). Ideally, a palliative endpoint is relevant to the majority of the target sample, and thus likewise to patients with short treatment duration, given that we may expect a palliative effect. The responsiveness of CBR to the effects of short treatments is limited by the complex structure of this algorithm.

CBR discriminated the QoL scores most strongly before starting chemotherapy. In line with the early study by Hoffman and Glimelius [5], CBR reflected mainly physical domains and did not capture the patients' perception of treatment burden. When evaluating a palliative treatment, outcomes complementary to pain, KPS and weight are important: the concept of CBR is too limited to reflect patients' perception. A clinically appealing feature, that CBR requires a minimal time period to qualify, can similarly be applied to PROs. The same applies to the time period the patients spend in the defined health state until deterioration.
The patients assessed their pain not only daily, as part of the documentation for CBR, but additionally weekly, or fortnightly, on a separate form that they completed at the hospital. The patient-rated indicators were less prone to missing data than the factors of CBR. The larger assessment intervals of the indicators were sufficiently small to capture the decisive changes relevant for the evaluation of a CBR. Overall, within this international phase III trial, the information derived from CBR was well covered and complemented by a few simple QoL indicators, without taking into account changes in analgesic consumption, performance status, or weight. Thus, our findings are equally relevant to phase III trials in other gastrointestinal cancer sites.

Depending on the QoL domain or symptom and time point of assessment, an improvement in QoL under single-agent gemcitabine has been reported in 2 phase II and 2 phase III trials, whereas 2 phase III trials showed either no change or a worsening [6]. Our working hypothesis was that the different time schedules used in these trials contributed to these inconsistent findings. In the present trial, QoL scores indicated consistent time effects also in patients receiving only few chemotherapy cycles [6]. It remains a matter of interpretation, whether the palliation as indicated by the patients was caused by a very brief anti-tumor effect by chemotherapy or by the conditions of the situation itself, for example, receiving antitumor treatment, supportive care, or more steroids than before [6].

To minimize the potential bias associated with early withdrawal from study treatment and to investigate patients' underlying trajectories of palliation, we have chosen shorter assessment intervals than in previous trials. In addition to the diaries used for the CBR evaluation, a comprehensive QoL assessment was not feasible. Our findings need to be interpreted in regard to the properties of the indicators used for this investigation. Assuming a comparable responsiveness to disease and treatment factors [20], these findings are relevant for other PROs, especially standard QoL measures.

In conclusion, the patient-rated indicators were less prone to missing data than CBR. CBR was associated with survival and mainly reflected physical domains. Within phase III chemotherapy trials for advanced gastrointestinal cancer, CBR can be replaced by a PRO evaluation, without losing substantial information, yet gaining complementary information.

\section{Acknowledgments}

We thank Susanne Cina for central data management and trial coordination. The following centers and investigators participated in this study: Switzerland: Aarau (C. Caspar, W. Mingrone); Basel (R. Herrmann, L. Jost, A. Lohri, C. Ludwig); Berne (M. Borner, D. Rauch); Chur (F. Egli); Geneva (A. Roth); Lausanne (J. Bauer, R. Popescu); St. Gallen (D. Köberle, R. Morant, T. Ruhstaller); Ticino (M. Bonomo, P. Saletti, C. Sessa); Zurich (H. Adam, L. Widmer, B. Pestalozzi); Austria: Feldkirch (A. Lang); Vienna (W. Scheithauer, J. Schüller); Finland: Oulo (T. Turpeen- 
niemi-Hujanen); Tampere (P. Kellokompu-Lehtinen); Turku (S. Pyrhönen); Germany: Dresden (C.H. Köhne, G. Kornek); Hungary: Budapest (G. Bodoky, K. Tamas); Israel: Petach Tikva (S. Stemmer); Tel-Aviv (A. Figer, M. Inbar); Italy: Milano (E. Bajetta); Napoli (P. Comella); Sweden: Uppsala (B. Glimelius). Financial support for the clinical trial was provided by Roche Pharma Switzerland, Eli Lilly Switzerland, the Swiss Federal Government and the Swedish Cancer Society.

\section{Disclosure Statement}

The authors indicate no potential conflicts of interest.

\section{References}

1 Booth CM, Ohorodnyk P, Eisenhauer EA: Call for clarity in the reporting of benefit associated with anticancer therapies. J Clin Oncol 2009;27:e213-14.

$\checkmark 2$ Rothenberg ML, Moore MJ, Cripps MC, et al.: A phase II trial of gemcitabine in patients with 5-FU-refractory pancreas cancer. Ann Oncol 1996; 7:347-353.

$\checkmark 3$ Burris HA 3rd, Moore MJ, Andersen J, et al.: Improvements in survival and clinical benefit with gemcitabine as first-line therapy for patients with advanced pancreas cancer: A randomized trial. J Clin Oncol 1997;15:2403-2413.

$\checkmark 4$ Gelber RD: Gemcitabine for pancreatic cancer: How hard to look for clinical benefit? An American perspective. Ann Oncol 1996;7:335-337.

$\checkmark 5$ Hoffman K, Glimelius B: Evaluation of clinical benefit of chemotherapy in patients with upper gastrointestinal cancer. Acta Oncol 1998;37:651659.

6 Bernhard J, Dietrich D, Scheithauer W, et al.: Clinical benefit and quality of life in patients with advanced pancreatic cancer receiving gemcitabine plus capecitabine versus gemcitabine alone: A randomized multicenter phase III clinical trial--SAKK 44/00-CECOG/PAN.1.3,001. J Clin Oncol 2008;26:3695-3701.

7 Fayers P: MRC quality of life studies using a daily diary card--practical lessons learned from cancer trials. Qual Life Res 1995;4:343-352.

8 Ohorodnyk P, Eisenhauer EA, Booth CM: Clinical benefit in oncology trials: Is this a patient-centred or tumour-centred end-point? Eur J Cancer 2009; 45:2249-2252.
9 Koeberle D, Saletti P, Borner M, et al.: Patientreported outcomes of patients with advanced biliary tract cancers receiving gemcitabine plus capecitabine: A multicenter, phase II trial of the Swiss Group for Clinical Cancer Research. J Clin Oncol 2008;26:3702-3708.

10 Herrmann R, Bodoky G, Ruhstaller T, et al.: Gemcitabine plus capecitabine compared with gemcitabine alone in advanced pancreatic cancer: A randomized, multicenter, phase III trial of the Swiss Group for Clinical Cancer Research and the Central European Cooperative Oncology Group. J Clin Oncol 2007;25:2212-2217.

11 Fishman B, Pasternak S, Wallenstein SL, et al.: The Memorial Pain Assessment Card. A valid instrument for the evaluation of cancer pain. Cancer 1987;60:1151-1158

12 Bernhard J, Maibach R, Thurlimann B, et al.: Patients' estimation of overall treatment burden: Why not ask the obvious? J Clin Oncol 2002;20:6572

13 Butow P, Coates A, Dunn S, et al.: On the receiving end. IV: Validation of quality of life indicators. Ann Oncol 1991;2:597-603.

14 Hürny C, Bernhard J, Coates A, et al.: Responsiveness of a single-item indicator versus a multi-item scale: Assessment of emotional well-being in an international adjuvant breast cancer trial. Med Care 1996;34:234-248.

15 Hürny C, Bernhard J, Bacchi M, et al.: The Perceived Adjustment to Chronic Illness Scale (PACIS): A global indicator of coping for operable breast cancer patients in clinical trials. Swiss Group for Clinical Cancer Research (SAKK) and the International Breast Cancer Study Group (IBCSG). Support Care Cancer 1993;1:200-208.

16 Bernhard J, Hürny C, Maibach R, et al.: Quality of life as subjective experience: Reframing of perception in patients with colon cancer undergoing radical resection with or without adjuvant chemotherapy. Swiss Group for Clinical Cancer Research (SAKK). Ann Oncol 1999;10:775-782.

17 Roth AD, Fazio N, Stupp R, et al.: Docetaxel, cisplatin, and fluorouracil; docetaxel and cisplatin; and epirubicin, cisplatin, and fluorouracil as systemic treatment for advanced gastric carcinoma: A randomized phase II trial of the Swiss Group for Clinical Cancer Research. J Clin Oncol 2007;25: 3217-3223.

18 Borner MM, Bernhard J, Dietrich D, et al.: A randomized phase II trial of capecitabine and two different schedules of irinotecan in first-line treatment of metastatic colorectal cancer: efficacy, quality-of-life and toxicity. Ann Oncol 2005;16: 282-288.

19 Zabora J, BrintzenhofeSzoc K, Curbow B, et al.: The prevalence of psychological distress by cancer site. Psychooncology 2001;10:19-28.

20 Bernhard J, Sullivan M, Hurny C, et al.: Clinical relevance of single item quality of life indicators in cancer clinical trials. Br J Cancer 2001;84:11561165.

21 Sloan JA, Dueck A: Issues for statisticians in conducting analyses and translating results for quality of life end points in clinical trials. J Biopharm Stat 2004;14:73-96. 